\title{
Deserved attention for acute kidney injury after major trauma
}

\author{
Ryan W. Haines ${ }^{1,2^{*}} \mathbb{D}$, Anatole Harrois ${ }^{3}$, John R. Prowle ${ }^{1,2,4}$, Signe Søvik ${ }^{5,6}$ and Sigrid Beitland ${ }^{7,8}$
}

๑ 2019 Springer-Verlag GmbH Germany, part of Springer Nature

\section{Initial correspondence from Drs. Haines, Harrois, and Prowle}

Dear Editor,

The systematic review of acute kidney injury (AKI) after trauma by Søvik et al. [1] adds much-needed data on the incidence and outcomes of trauma-associated AKI. The authors reported a pooled incidence of AKI of $24 \%$ across 24 studies including over 25,000 patients. These results duplicate those of a recently published meta-analysis of AKI in trauma by Haines et al. [2]. Furthermore, data on AKI in 3111 patients from a French multicentre trauma registry has now been published by Harrois et al. [3] where an independent association between AKI and mortality persisted. When included amongst studies reporting adjusted odds ratios of death in trauma-ICU patients with AKI, the association with mortality is convincing, Fig. 1.

Søvik et al. [1] suggest that AKI recovery in survivors is good (96\% of patients recovering). We disagree with this interpretation. Firstly, differing definitions of kidney recovery are simplistic; successful weaning from ongoing renal replacement therapy (RRT), in the small group who require it, and non-return to baseline creatinine are disparate, non-comparable outcomes. Secondly, in the largest study in the pooled analysis, which incorporated a return to baseline creatinine definition of recovery, trauma-AKI was associated with non-recovery. Finally, muscle wasting during critical illness is near universal, resulting in a confounding effect of decreased muscle volume lowering creatinine generation, so that even with a return to baseline creatinine suggesting recovery, chronic

*Correspondence: r.haines@qmul.ac.uk

${ }^{1}$ Adult Critical Care Unit, The Royal London Hospital, Barts Health NHS Trust, Whitechapel Road, London E1 1BB, UK

Full author information is available at the end of the article renal injury may have occurred. Given these considerations, we believe the true extent of recovery of AKI after trauma remains unknown and is unlikely to be a self-limiting phenomenon.

\section{Reply from Drs. Søvik and Beitland}

We would like to thank Haines, Harrois and Prowle for thoughtful remarks on our recent review [1], pointing at important issues concerning long-term outcomes after acute kidney injury (AKI) in trauma patients admitted to the ICU. Haines et al. [2] confirm our finding that post-traumatic AKI is common, and add valuable new information about the independent association between AKI and increased mortality. However, the authors disagree with our interpretation of findings regarding AKI recovery.

In our review, pooled analysis of data revealed that renal recovery occurred in $96 \%$ of patients. Though this implies an optimistic prognosis after AKI in trauma patients, we elaborate in our discussion how this conclusion is fraught with uncertainties. We underline problems introduced by short follow-up times, lack of standardized time points for evaluating kidney function, and variable definitions of renal recovery. We also mention that an episode of AKI has been associated with increased risk of chronic kidney disease in other critically ill patients [4].

Haines et al. [2] expand this important discussion and mention the difficulties in pooling data on renal recovery when heterogeneous definitions are used. They correctly state that the largest study in our pooled analysis reported complete renal recovery of $51 \%$, and that critical illness muscle wasting will impact on creatinine-based renal criteria.

We agree that our reported result regarding renal recovery has limitations, and again conclude that there is a clear need for standards of reporting in AKI, including a consensus definition of renal recovery.

\section{实 Springer}




\begin{tabular}{|c|c|c|}
\hline Study & log[Odds Ratio] & SE \\
\hline Shashaty & 0.7 & 0.1723 \\
\hline Bihorac & 1.1 & 0.2904 \\
\hline Bagshaw & 0.6 & 0.0977 \\
\hline Stewart & 1.6 & 0.2213 \\
\hline Podoll & 1.2 & 0.3246 \\
\hline Haines & 0.9 & 0.2388 \\
\hline Harrois & 0.7 & 0.2038 \\
\hline
\end{tabular}

Random effects model

Heterogeneity: $I^{2}=74 \%, \tau^{2}=0.1061, p<0.01$

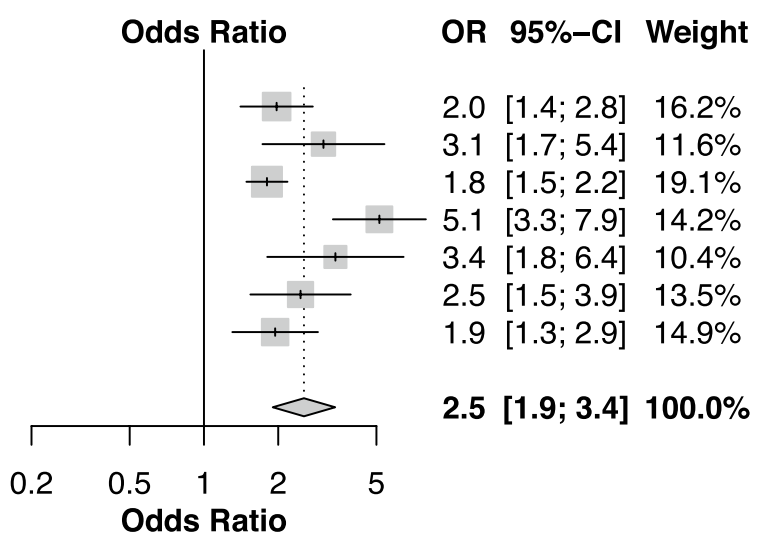

Fig. 1 Random-effects meta-analysis of the effect of acute kidney injury diagnosis on short-term survival in studies with multivariable adjustment

\begin{abstract}
Author details
${ }^{1}$ Adult Critical Care Unit, The Royal London Hospital, Barts Health NHS Trust, Whitechapel Road, London E1 1BB, UK. ${ }^{2}$ William Harvey Research Institute, Queen Mary University of London, London, UK. ${ }^{3}$ Department of Anesthesiology and Critical Care, Université Paris Sud, Université Paris Saclay, Assistance Publique-Hopitaux de Paris (AP-HP), Bicêtre Hopitaux Universitaires Paris Sud, 78 rue du Général Leclerc, F-94275 Le Kremlin Bicêtre, France. ${ }^{4}$ Department of Renal Medicine and Transplantation, The Royal London Hospital, Barts Health NHS Trust, Whitechapel Road, London E1 1BB, UK. ${ }^{5}$ Department of Anaesthesia and Intensive Care, Akershus University Hospital, Lørenskog, Norway. ${ }^{6}$ Institute of Clinical Medicine, Faculty of Medicine, University of Oslo, Oslo, Norway. ${ }^{7}$ Renal Research Group Ullevål, Institute of Clinical Medicine, Faculty of Medicine, University of Oslo, Oslo, Norway. ${ }^{8}$ Division of Emergencies and Critical Care, Department of Anaesthesiology, Oslo University Hospital Ullevål, Oslo, Norway.
\end{abstract}

\section{Compliance with ethical standards}

\section{Conflicts of interest}

On behalf of all authors, the corresponding author states that there is no conflict of interest.

\section{Publisher's Note}

Springer Nature remains neutral with regard to jurisdictional claims in published maps and institutional affiliations.
Accepted: 25 March 2019

Published online: 23 April 2019

\section{References}

1. Søvik S, Isachsen MS, Nordhuus KM et al (2019) Acute kidney injury in trauma patients admitted to the ICU: a systematic review and metaanalysis. Intensive Care Med 30:581. https://doi.org/10.1097/CCM.0b013 e3182227a59

2. Haines RW, Fowler AJ, Kirwan CJ, Prowle JR (2019) The incidence and associations of acute kidney injury in trauma patients admitted to critical care: A systematic review and meta-analysis. J Trauma Acute Care Surg 86:141-147

3. Harrois A, Soyer B, Gauss T, Hamada S, Raux M, Duranteau J, Group T (2018) Prevalence and risk factors for acute kidney injury among trauma patients: a multicenter cohort study. Crit Care 22:344

4. Chawla LS, Eggers PW, Star RA, Kimmel PL (2014) Acute kidney injury and chronic kidney disease as interconnected syndromes. N Engl J Med 371:58-66. https://doi.org/10.1056/NEJMra1214243 